\section{CRITERIOS DE SÍNDROME METABÓLICO Y OBESIDAD ABDOMINAL PARA POBLACIONES ANDINAS}

\author{
METABOLIC SYNDROME AND ABDOMINAL \\ OBESITY CRITERIA FOR ANDEAN \\ POPULATIONS
}

\section{Offdan Narvaez-Guerra' ${ }^{1,2, a}$, Karela Herrera-Enriquez ${ }^{1,2, a}$}

Sr. Editor. Hemos leído con interés el estudio de Ninatanta-Ortiz et al. realizado en dos distritos urbanos de la región Cajamarca, localizados a 2388 y 2750 msnm respectivamente, en 1427 participantes, que incluyeron a estudiantes de secundaria, universitarios, y madres de estudiantes de primaria, donde reportan que la frecuencia de síndrome metabólico (SM) fue 3,2\%, $1,6 \%$, y $23,5 \%$ respectivamente; además de comparar sus hallazgos con los de países vecinos ${ }^{(1)}$.

En el año 2004 se publicó el reporte del Panel de Tratamiento en Adultos del Programa Nacional de Educación en Colesterol (NCEP/ATPIII) que definió los criterios del SM en adultos, dentro de cuyos componentes consideró como criterios a la circunferencia abdominal $>102 \mathrm{~cm}$ en hombres y > $88 \mathrm{~cm}$ en mujeres (2). Ninatanta-Ortiz et al. ${ }^{(1)}$ utilizaron estos criterios para el diagnóstico de SM en adultos de su estudio, los cuales se encuentran actualmente vigentes para el diagnóstico de SM en adultos norteamericanos.

La nueva definición mundial de SM determinada por la Federación Internacional de Diabetes (IDF) considera a la obesidad abdominal como un criterio esencial, teniendo en cuenta algunas diferencias étnicas en los puntos de corte ${ }^{(3)}$. Sin embargo, este organismo establece que el criterio para obesidad abdominal en poblaciones sudamericanas no ha sido determinado, y que para tal fin se debe utilizar los criterios establecidos para sujetos de Asia del Sur (circunferencia abdominal $\geq$ $90 \mathrm{~cm}$ en hombres $y \geq 80 \mathrm{~cm}$ en mujeres) ${ }^{(3)}$. Es decir, las principales definiciones internacionales de SM no han establecido los puntos de corte de obesidad abdominal para ser utilizadas en poblaciones latinoamericanas o sudamericanas.

\footnotetext{
Departamento de Medicina Preventiva, Centro de Medicina Ocupacional Integral CEMOIN, Arequipa, Perú

2 Instituto de Investigación Cardiológico PREVENCION, Universidad Católica de Santa María, Arequipa, Perú

a Médico cirujano

Recibido: 24/12/2016 Aprobado: 11/01/2017 En línea: 27/02/2017
}

Citar como: Narvaez-Guerra O, Herrera-Enriquez K. Criterios de síndrome metabólico y obesidad abdominal para poblaciones andinas [carta]. Rev Peru Med Exp Salud Publica. 2017;34(1):147-8. doi: 10.17843/rpmesp.2017.341.2721
Debemos mencionar que la revista de la Asociación Americana de Diabetes (ADA) ha publicado los resultados del estudio PREVENCION, realizado en una muestra heterogénea de 1448 andinos hispánicos de Arequipa (2326 de altitud), en quienes se compararon la relación de circunferencia abdominal, enfermedad vascular subclínica (grosor miointimal carotídeo), y enfermedad cardiovascular manifiesta, encontrándose que los puntos de corte de circunferencia abdominal $>97$ $\mathrm{cm}$ en hombres y $>87 \mathrm{~cm}$ en mujeres caracterizaban, con mayor sensibilidad que los criterios NCEP/ATPIII, a los portadores de SM ${ }^{(4)}$, por lo que consideramos que estos criterios serían de mayor utilidad para la muestra del estudio de Ninatanta-Ortiz et al. al haber sido realizados en poblaciones, alturas, y condiciones similares.

La importancia de una correcta caracterización de la circunferencia abdominal reside en que es un indicador único de distribución de grasa corporal que brinda valiosa información en consultorio, además, permite identificar a los pacientes que deben ser evaluados para descartar la presencia de dislipidemia y/o hiperglicemia, al ser un marcador sensible de riesgo cardiometabólico ${ }^{(5)}$.

En conclusión, los criterios vigentes de SM del NCEP/ ATP III e IDF no toman en cuenta la diversidad étnica de poblaciones latinoamericanas. Estudios realizados sobre la prevalencia de SM en Perú deberían usar los criterios que han sido demostrados como sensibles para $\mathrm{SM}$ en tales poblaciones, y en la medida de lo posible, realizar una comparación con los criterios NCEP/ATPIII e IDF y de esta manera analizar la variabilidad existente.

Contribuciones de los autores: ONG y KHE han participado en la concepción del artículo, su redacción, revisión crítica del artículo, y aprobación de la versión final.

Fuentes de financiamiento: autofinanciado.

Conflictos de interés: los autores declaran no tener conflictos de intereses.

\section{REFERENCIAS BIBLIOGRÁFICAS}

1. Ninatanta-Ortiz JA, Núñez-Zambrano LA, García-Flores SA, Romaní Romaní F. Frecuencia de síndrome metabólico en residentes de una región andina del Perú. Rev Peru Med Exp Salud Publica. 2016;33(4):640-50. doi: 10.17843/ rpmesp.2016.334.2546.

2. Grundy SM, Brewer HB, Cleeman JI, Smith SC, Lenfant C. Definition of Metabolic Syndrome: Report of the National Heart, Lung, and Blood Institute/American Heart Association Conference on Scientific Issues Related to Definition. Circulation. 2004;109(3):433-8. doi: 10.1161/01. CIR.0000111245.75752.C6.

3. International Diabetes Federation (IDF) [Internet]. The IDF consensus worldwide definition of the metabolic syndrome; 2006 [Citado el 20 de diciembre de 2016] Disponible en: http://www.idf.org/webdata/docs/IDF_Meta_def_final.pdf 
4. Medina-Lezama J, Pastorius CA, Zea-Diaz H, Bernabe-Ortiz A, Corrales-Medina F, Morey-Vargas OL, et al. Optimal Definitions for Abdominal Obesity and the Metabolic Syndrome in Andean Hispanics: The PREVENCION Study. Diabetes Care. 2010;33(6):1385-8. doi: 10.2337/dc09-2353.

5. Klein S, Allison DB, Heymsfield SB, Kelley DE, Leibel RL, Nonas C, et al. Waist Circumference and Cardiometabolic Risk. A Consensus Statement from Shaping America's Health: Association for Weight Management and Obesity Prevention; NAASO, The Obesity Society; the American Society for Nutrition; and the American Diabetes Association. Diabetes Care. 2007;30(6):1647-52. doi: 10.2337/dc07-9921.

Correspondencia: Offdan Narváez Guerra

Dirección: Avenida Victor Andrés Belaúnde B8 202, Umacollo, Arequipa, Arequipa, Perú.

Teléfono: (054) 968954571

Correo electrónico:offdannarvaez@gmail.com

\section{CRITERIOS DE SÍNDROME METABÓLICO Y OBESIDAD ABDOMINAL PARA POBLACIONES ANDINAS-RÉPLICA}

\section{METABOLIC SYNDROME AND ABDOMINAL OBESITY CRITERIA FOR ANDEAN POPULATIONS - REPLY}

\author{
Juana Aurelia Ninatanta-Ortiz ${ }^{1, a}$, Franco Romaní \\ Romaní ${ }^{2, b}$
}

\begin{abstract}
Sr. Editor. Consideramos importante la observación realizada respecto a la selección de criterios para síndrome metabólico (SM) en poblaciones latinoamericanas, y específicamente en poblaciones andinas. Realizamos la selección de los criterios ATP III debido a su uso frecuente en estudios de prevalencia de SM en población andina (1), incluyendo el estudio PREVENCIÓN realizado en pobladores de la ciudad de Arequipa ${ }^{(2)}$; de esta forma es posible comparar resultados con otras poblaciones de América Latina (andinas o no) ${ }^{(3)}$; otro aspecto importante es que los criterios ATP III, a diferencia de los criterios IDF, no requieren como componente obligatorio la obesidad abdominal ${ }^{(4)}$.
\end{abstract}

Se han dado recomendaciones de cortes de circunferencia de cintura para poblaciones europeas, el consenso publicado el $2009{ }^{(4)}$ recomienda para América Latina usar como punto de corte los valores obtenidos en poblaciones del Sur de Asia ( $\geq 90 \mathrm{~cm}$ en varones $y \geq 80 \mathrm{~cm}$ en mujeres), sin embargo, dichos cortes no contemplan el aspecto de la etnicidad mixta, característica común en las zonas urbanas de los andes peruanos. A nivel nacional, Medina-Lezama et al. recomiendan el uso de cortes de circunferencia de cintura específicos por etnia, para ello modifican los criterios AHA/NHLBI planteando para el componente de obesidad abdominal los cortes $>97 \mathrm{~cm}$ en varones y $>87 \mathrm{~cm}$ en mujeres ${ }^{(5)}$, sin embargo dichos cortes fueron obtenidos de un estudio transversal, y no son representativos para todas las poblaciones mestizas de los andes.

La aplicación de diferentes criterios de SM genera estimaciones de prevalencia que varian entre 23,5 a $34,3 \%$ en madres de estudiantes de primaria, y entre 1,6 y $10,2 \%$ en estudiantes universitarios. Los criterios IDF (International Diabetes Federation) permiten estimar mayores prevalencias que los criterios ATP III (Third Adult Treatment Panel) y AHA/NHLBI (American Heart Association/National Heart, Lung, and Blood Institute), debido a que criterios IDF usan menores puntos de corte para circunferencia de cintura ( $\geq 90 \mathrm{~cm}$ en varones y $\geq 80 \mathrm{~cm}$ en mujeres), y para glucosa sérica en ayunas ( $\geq 100 \mathrm{mg} / \mathrm{dL})$.

Usando los criterios AHA/NHLBI modificados (5) encontramos una prevalencia de $26,9 \%$ en madres y $2,6 \%$ en universitarios, estimaciones cercanas a lo reportado en nuestro estudio. La prevalencia de SM usando los criterios ATP III, considerando como corte sugerido para circunferencia de cintura $>97 \mathrm{~cm}$ en varones y $>87 \mathrm{~cm}$ en mujeres, es de $25,0 \%$ en madres y $2,0 \%$ en universitarios (Tabla 1 ).

La evidencia disponible no brinda valores referenciales para establecer puntos de corte adaptados para diversas poblaciones andinas de América del Sur. En dichos ámbitos el mestizaje y la etnicidad mixta podrían afectar los valores mínimos y máximos de la circunferencia de cintura. En conclusión, el uso de diferentes definiciones operacionales afecta en forma importante las estimaciones de SM en poblaciones de los andes $u$ otras poblaciones de Latinoamérica. La estimación de SM usando criterios ATP III permite la comparación de nuestros resultados con otros estudios realizados en la región, dichas estimaciones son concordantes con los criterios AHA/NHLBI clásicos y modificados.
Escuela Académico Profesional de Enfermería, Facultad Ciencias de la Salud, Universidad Nacional de Cajamarca. Cajamarca, Perú

2 Oficina General de Investigación y Transferencia Tecnológica, Instituto Nacional de Salud. Lima, Perú.

a Enfermera, doctora en Ciencias de Enfermería, ${ }^{\text {b }}$ médico cirujano Recibido: 27/01/2017 Aprobado: 08/02/2017 En línea: 27/02/2017
Citar como: Ninatanta-Ortiz JA, Romaní Romaní F. Criterios de síndrome metabólico y obesidad abdominal para poblaciones andinas - Réplica [carta]. Rev Peru Med Exp Salud Publica. 2017;34(1):148-9. doi: 10.17843/ rpmesp.2017.341.2720 